\title{
Relationship between fractional excretion of magnesium and renal histology in glomerulonephritis
}

\begin{abstract}
Progressive pediatric glomerularnephroses can lead to tubulointerstitial fibrosis (TIF) and chronic kidney disease (CKD). Fractional excretion of magnesium (FEMg) is a sensitive tubular function test (Ref). FEMg correlates positively with TIF (Ref). The purpose of the study was to correlate FEMg with renal histolopathology in pediatric glomerular nephrosis (GN). Sixty three cases, between 1-12 years, of biopsy diagnosed GN were included purposively in this cross sectional study from November 2008 to April 2010. Cases were taken from Department of Paediatric Nephrology, Dhaka Shishu (Children) Hospital and Department of Paediatric Nephrology, National Institute of Kidney Diseases \& Urology, Sher-e-Bangla Nagar, Dhaka, Bangladesh. Minimal change disease (MCD) was most common at $27 \%$ and remaining $73 \%$ were other than MCD. The mean FEMg in MCD was normal $(1.810 .34 \%)$ but in other than MCD as a group it was significantly elevated $(7.60$ $\pm 19.23 \% ; \mathrm{p}<0.001)$. and significantly elevated separately in diffuse mesangial proliferative glomerulonephritis $(3.96 \pm 1.06 \% ; \mathrm{p}<0.001)$, focal segmental glomerulosclerosis $(9.99 \pm$ $4.45 \% ; \mathrm{p}<0.01)$, focal segmental proliferative glomerulonephritis $(4.63 \pm 2.29 \% ; \mathrm{p}<0.05)$ and crescentic glomerulonephritis $(17.744 .93 \%$; $\mathrm{p}<0.01)$
\end{abstract}

Keywords: fractional excretion of magnesium, glomerulonephritis, tubular interstitial disease
Volume 5 Issue 4 - 2016

\author{
Mohammed Maruf-ul-Quader,' Mohammed \\ Hanif, ${ }^{2}$ Shireen Afroz, ${ }^{2}$ Mohammed Anwar \\ Hossain Khan, ${ }^{3}$ Jubaida Rumana ${ }^{4}$ \\ 'Department of Child Health, Chittagong Medical College, \\ Bangladesh \\ ${ }^{2}$ Department of Pediatric Nephrology, Dhaka Shishu (Children) \\ Hospital \& Bangladesh Institute of Child Health, Bangladesh \\ ${ }^{3}$ Department of Pediatric Nephrology, National Institute of \\ kidney Diseases and Urology, Bangladesh \\ ${ }^{4}$ Department of Pediatrics \& Neonatology, Bangladesh Institute \\ of Health Sciences General Hospital, Bangladesh
}

\author{
Correspondence: Mohammed Maruf-ul- Quader,Assistant \\ Professor, Division of Pediatric Nephrology, Department \\ of Child Health, Chittagong Medical College, Chittagong, \\ Bangladesh, Tel +88019/ I223237, \\ Emailmarufulquader@yahoo.com
}

Received: August 02, 2016 | Published: October 26, 2016

\section{Introduction}

Numerous inflammatory and non inflammatory diseases affect the glomerulus and lead to alteration in glomerular permeability, structure \& function. Glomerulonephritis $(\mathrm{GN})$ is a generic term for several diseases and a histopathologic term signifying inflammation \& proliferation of cells within the glomerulus. ${ }^{1}$ It may be primary, restricted to kidney or may be part of a multisystem diseases. Clinical evaluation includes assessment of proteinuria, hematuria, presence or absence of renal insufficiency and presence or absence of hypertension and ultimately diagnosed with renal histology. ${ }^{2,3}$ Glomerular diseases may have an indolent course or begin abruptly leading to acute or rapidly progressive glomerulonephritis. Chronic glomerulonephritis implies continuing glomerular injury that frequently leads to glomerular destruction and end stage renal failure. Glomerulus may be injured by several mechanism in different diseases but produce limited number even same histopathologic changes. The full assessment of a renal histology requires light microscopy, electron microscopy and immunofluorescence. ${ }^{4}$ Proliferation of glomerular cells occur in most form of glomerulonephritis, commonly involves endothelial and mesangial cells and sometimes there may have crescent and sclerosis. ${ }^{5}$ Abnormalities are not confined only to glomerulus , tubulointerstitial changes attend almost all forms of progressive glomerular and vascular injury. In most cases of glomerulonephritis long term course lead to chronic renal failure and characterized by tubular atrophy, interstitial fibrosis. ${ }^{6}$

In children the most common presentation of glomerulonephritis is nephrotic syndrome. ${ }^{7}$ Histologically minimal change disease (MCD) is the commonest $76.4 \%$, other histological pattern are mesangial proliferative glomerulonephritis (MesPGN) 2.3\%, membranoproliferative glomerulo nephritis (MPGN) $7.5 \%$, focal segmental glomerulosclerosis (FSGS) 6.9\%, membranous nephropathy(MN) $1.5 \% .8$ Other pattern of glomerulonephritis in pediatric patients are lupus nephritis, IgA nephropathy, Henoch Schonlein purpura nephritis or isolated hematuria and or proteinuria. In NS, 30-40\% steroid resistant MCD develops end stage renal disease by 5 years. Similarly MesPGNsz (50\%), MPGN (20-30\%), FSGS $(21 \%)$ and $\mathrm{MN}(7.3 \%)$ progress to end stage renal disease within 5 years of age. ${ }^{9-13}$

Two patterns of clinicopathologic subsets have been generally delineated in nephrosis namely 1) a benign clinicopathologic entity associated with no tubulointerstitial disease such as minimal change lesion or mild mesangial proliferative glomerulonephritis 2) A progressive clinicopathologic disease associated with tubulointerstitial lesion such as that observed in focal segmental glomerulosclerosis, a moderate to diffuse mesangial proliferative glomerulonephritis and membranoproliferative glomerulonephritis. The clinical course in the former is usually associated with a self limited disease without renal diseases progression where as in the latter progressive renal disease is destined for chronic renal insufficiency and end stage renal disease. Tubular function testing i.e. may predict end stage renal disease. FEMg can reflect tubular function FEMg $<2.2 \%$ indicates intact tubular function whereas elevated FEMg indicates tubular damage. The greater the FEMG the greater the magnitude of TIF.

\section{Materials and methods}

In this cross sectional study 63 patients, between 1-12 years of age, of histologically diagnosed glomerulonephritis were included. The study was performed in Department of Pediatric Nephrology, Dhaka Shishu Hospital \& National Institute of Kidney Diseases \& Urology, Sher-e-Bangla Nagar, Dhaka in the period of November 2008 to April 2010. Informed written consent was obtained from the legal guardian. Reassurance was given to the guardian regarding investigation. Clearance has been taken from the ethical committee. No study was cost was paid by study subjects. Biochemical indices and renal biopsy report were noted. Diuretics and aminoglycosides, they were stopped 
24 hours and 48 hours prior to collection of serum and urine [Q: is this long enough?]. $10 \mathrm{ml}$ urine samples were centrifuged and stored in refrigerator. Serum creatinine and magnesium, spot urinary creatinine and magnesium were measured. The MG method was used on the Dimension ${ }^{\circledR}$ (Siemens, address) Clinical Chemistry System. FEMg was then calculated using the following formula:

$$
\text { FEMg }=\frac{(\text { Urine magnesium } / \text { plasma magnesium })}{(\text { Urinecreatinine } / \text { plasmacreatinine })} \times 100
$$

(Normal value of FEMg: $2.2 \%$ or less)

Light microscopy and direct immunofluorescent examinations were reviewed by a histopathologist blinded to FEMg (Table 1). Data were collected, compiled and analyzed by using SPSS version 12 . Independent $-t$ test was used as the test for significance. $P$ value of $<0.05$ was considered statistically significant.

\section{Results}

FEMg for each diagnosis is given in Table 1. FEMg in MCD was normal and taken as standard. FEMg in MesPGN and MPGN was found increased but not significant (Figure 1, Table 2\&3). In case of D-MesPGN, FSGN, FSGS and crescentic glomerulonephritis FEMg was increased and statistically significant. Chronic sclerosing GN and IgA Nephropathy were one each, so these are not mentioned in the table.

Table I Distribution of patients with glomerulonephritis by clinical diagnosis

\begin{tabular}{lll}
\hline Clinical diagnosis of the patient & Frequency & Percentage (\%) \\
\hline Nephrotic syndrome (NS) Ist attack & 22 & 34.92 \\
Frequent relapse nephrotic syndrome (FRNS) & 17 & 26.98 \\
Steroid dependent nephrotic syndrome (SDNS) & 8 & 12.7 \\
Steroid resistant nephrotic syndrome (SRNS) & 1 & 1.59 \\
Nephrotic syndrome with renal failure & 2 & 3.17 \\
HSP with nephritic-nephrotic syndrome & 1 & 1.59 \\
SLE with Nephritis & 4 & 6.35 \\
AGN with ARF & 6 & 9.52 \\
Acute renal failure & 1 & 1.59 \\
Chronic kidney disease & 1 & 1.59 \\
Total & 63 & 100 \\
\hline
\end{tabular}

Table 2 Relation of FEMg with renal histopathology of the patient

\begin{tabular}{llllll}
\hline Renal histopathology of the patient & No. & Mean FEMg(\%) & ISD FEMg & t-value & P \\
\hline Minimal change disease (MCD) & 17 & 1.81 & 0.34 & - & - \\
Mesangial proliferative glomerulonephritis(MesPGN) & 4 & 3.5 & 1.08 & 3.07 & $>0.05, \mathrm{~ns}$ \\
Diffuse mesangial proliferative glomerulonephritis (D-MesPGN) & 13 & 3.96 & 1.06 & 3.96 & $<0.001, \mathrm{~s}$ \\
Focal segmental proliferative glomerulonephritis (FSGN) & 10 & 4.63 & 2.29 & 3.86 & $<0.05, \mathrm{~s}$ \\
Focal segmental glomerulosclerosis (FSGS) & 9 & 9.99 & 4.45 & 5.49 & $<0.01, \mathrm{~S}$ \\
Crescentic glomerulonephritis & 5 & 17.74 & 4.93 & 7.21 & $<0.01, \mathrm{~S}$ \\
Membranoproliferative glomerulonephritis(MPGN) & 3 & 13.97 & 4.28 & 4.91 & $\mathrm{~ns}$ \\
\hline
\end{tabular}

SD, standard deviation; S, significant; NS, not significant

Table 3 Comparison of FEMg between minimal change disease (MCD) and other than minimal change disease

\begin{tabular}{lllllll}
\hline Variable & Subject & Number & Mean & $\mathbf{I S D}$ & t-value & P \\
\hline \multirow{2}{*}{ Fractional excretion of magnesium } & MCD & 17 & 1.81 & 0.34793 & \multirow{2}{*}{6.95} & $<0.001, S$ \\
& Other than MCD & 46 & 7.6 & 19.234 & & \\
\hline
\end{tabular}

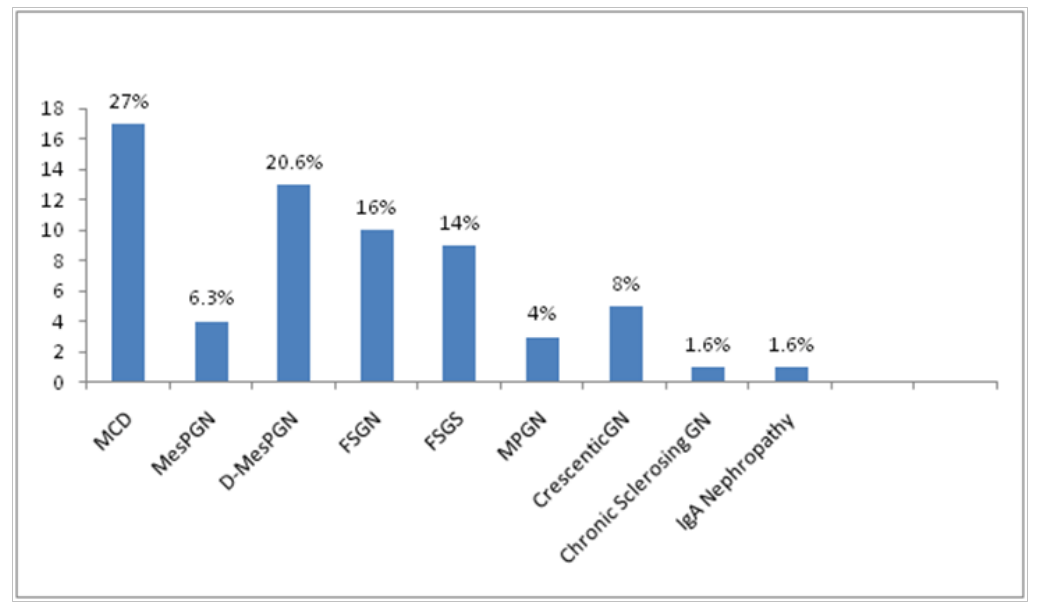

Figure I Types of histopathological findings found in biopsy.

Citation: Maruf-ul-Quader M, Hanif M, Afroz S, et al. Relationship between fractional excretion of magnesium and renal histology in glomerulonephritis.J Pediatr Neonatal Care. 2016;5(4):I I-I2. DOI: 10.15406/jpnc.2016.05.00I92 


\section{Discussion}

Disease incidence was similar to the study by Madani A et al., ${ }^{7}$ (66.26\%) and Al-Rasheed et al., ${ }^{14}$ (77\%). In this study FEMg was normal in MCD $(1.81+0.34 \%)$. In D-MesPGN $(3.96 \pm 1.06)$, FSGS $(9.99 \pm 4.45)$, crescentic GN (17.74 \pm 4.93$), \mathrm{FEMg}$ found abnormally elevated and statistically highly significant $(\mathrm{p}<0.01)$. In FSGN $(4.63 \pm 2.29) \mathrm{FEMg}$ also found elevated and significant $(\mathrm{p}<0.05)$ FSGS showed abnormally elevated FEMg in studies by Futrakul P; Deekajorndech T similar to this study. FSGN, crescentic GN and MPGN were not included in other studies; which are important cause of CKD. ${ }^{11-18}$ (Please discuss other studies which reference FEMg- the purpose of the study was not so much to survey disease incidence but rather to correlate FEMg with histopatholgic diagnosis) Tubulointerstitial injury is an invariant finding in the chronically diseased kidney, irrespective of the type of disease. ${ }^{19}$ FEMg appeared as a sensitive indicator of severity of renal insufficiency as showed in chronic renal failure patients of Nigeria. ${ }^{20-22}$

(I don't think the purpose of the study was to compare markers) Glomerular filtration rate, peretubular capillary blood flow, afferent arteriolar resistance were not assessed in this study which are important factor for tubular dysfunction or TIF due to limited resources but assessed in different study. ${ }^{23} \mathrm{FEMg}$ is normal in minimal change disease and abnormally elevated in other than minimal change disease which is statistically significant.

\section{Conclusion}

Fractional excretion of magnesium increases in other than minimal change disease and may be of value as a simple investigation in glomerulonephritis to assess disease severity.

\section{Acknowledgments}

None.

\section{Conflicts of interest}

Authors declare that there is no conflict of interest.

\section{References}

1. Watson R, Taylor MC, McGraw M. Disorders of the urinary system. In: Mclntosh N \& Smyth LR (Eds.), Forfar \& Arneil's Texbook of Pediatrics. 6th ed. Churchill Livingstone: Spain; 2003. 613-620 p.

2. Falk JR, Chaules J, Nachman HP. Primary Glomerular Disease. In Brenner MB (Ed.), Brenner and Rectors The Kidney. 7th ed. Elsevier, Philadelphia, USA; 2004. 1293 p.

3. Johnson JR. Introduction to Glomerular disease, Pathogenesis and classification. In: Feehally J \& Richard JJ (Eds.), Comprehensive clinica Nephrology. 3rd ed. Elsevier: Philadelphia, USA; 2007. 181-191 p.

4. Charles E, Alpers. The Kidney. In: Kumar, Abbas \& Fausto (Eds.) Robbins \& Cortan Pathologic basis of disease. 7th ed. Elsevier Saunders: Philadelphia, USA; 2005. 955-959 p.

5. Davis DI, Avner DI. Glomerular Disease. In: Kliegman, Behrman \& Stanton (Eds.), Nelson Text book of pediatrics. 7th ed. Elsevier: Philadelphia, USA; 2007. 2163-2167 p.
6. Idasiak-Piechocka, Krzymański M. The role of tubulo interstitial changes in progression of kidney failure in patient with chronic glomerulonephritis. Przegl Lek. 1996;53(5):443-453.

7. Madani A, Daryoush F, Esfehani TS, et al. Glomerular diseases in Iranian children: clinico-pathological correlation. Pediatr Nephrol. 2003;18(9):925-928.

8. ISKDC the Primary Nephrotic Syndrome in children Identification of patients with minimal change nephrotic syndrome from initial response to prednisane. J Pediatr. 1981;98(4):561-564.

9. Bagga A, Srivastava RN. Nephrotic syndrome Pediatric Nephrology. 5th ed. Jaypee Brothers Medical Publishers: New Delhi; 2011. 219-224 p.

10. Niaudut P, Boyer O. Idiopathic Neophrotic syndrome in children: Clinical Aspects. Pediatric Nephrology, 6th ed. Heidelberg: springer 2009. 667-702 p.

11. Futrakul P, Yanrudi S, Futrakul N, et al. Tubular function and tubulointerstitial Disease. American Journal of Kidney Diseases. 1999;33(5):886-891.

12. Deekajorndech T. Fractional Excretion of Magnesium in Systemic lupus Erythematososus. J Med Assoc Thai. 2005;88(6):743-745.

13. Futrakul N, Futrakul P. Prevention of End-stage renal disease: An Innovative strategy. Thailand, chulalongkorn university printing house; 2008. 2-31 p.

14. Al-Rasheed SA, Al-Mugeiren MM, Al-Salloum AA, et al. Childhood renal disease in Saudi Arabia. A clinico-pathological study of 167 cases. Int Urol Nephrol. 1996;28(5):607-613.

15. Srivastava T, Simon SD Alon US. High incedence of FSGS in nephrotic syndrome of childhood. Pediatr Nephrol. 1999;13:13-18.

16. Gianoglio B. From the IItalian registry of Pediatric renal biopsies Pediatr Nephrol. 1998;12:225.

17. Deekajorndech TA. Biomarker for Detecting Early Tubulointerstial Disease and ischaemia in Glomerulonephropathy. Renal Failure. 2007;29:1013-1017.

18. Futrakul N, Sila-asna M, Futrakul P. Therapeutic Strategy towards renal restoration in chronic kidney disease. Asian Biomedicine. 2007;1(1):33-43.

19. Nath KA. Tubulointerstial changes as a major determinant in the progression of renal damage. Am J Kidney Dis. 1992;20(1):1-17.

20. Oladipo OO, Onubi J, Awobusuyi O, et al. Fractional excretion of magnesium of chronic renal failure patients in Lagos, Nigeria. Niger Post grad Med J. 2003;10(3):131-134.

21. Futrakul N, Panichakul T, Sirisinha S, et al. Glomerular endothelial dysfunction in chronic kidney disease. Renal Fail. 2004;26(3):259-264.

22. Bhimma R, Adhikari M, Asharam K, et al. The spectrum of kidney disease (stage 2-5) in KwaZulu-Natal, South africa. Pediatr Nephrol. 2004;23(10):1841-1846.

23. Futrakul N, Yenrudi S, Sensirivatana R, et al. Peritubular capillary flow determines tubulointerstitial disease in idiopathic Nephrotic syndrome. Renal Fail. 2000;22(3):329-335. 